\title{
Therapeutic management of acute type A aortic intramural hematoma
}

\author{
Jue Yang ${ }^{1 \dagger}$, Changjiang Yu ${ }^{1+}$, Xin $\mathrm{Li}^{1 \dagger}$, Juntao Kuang ${ }^{1,2}$, Zerui Chen ${ }^{1}$, Fei Xiao ${ }^{1}$, Tucheng Sun ${ }^{1}$, \\ Miaoxian Fang ${ }^{1 *}$ and Ruixin Fan ${ }^{1 *}$
}

\begin{abstract}
Objectives: The proper therapeutic management for acute type A aortic intramural hematoma (IMH) is still controversial. The purpose of this study was to compare the outcomes following emergency surgery or conservative treatment for patients with this disease.

Methods: From January 2015 to December 2018, 124 consecutive patients were diagnosed with an acute type A aortic IMH and were included in this study. According to our surgical indications, they were divided into two groups: an operation group (OG) and a conservative treatment group (CG).

Results: Of 124 patients, 83 (66.9\%) patients accepted emergency surgery and 41 (33.1\%) patients accepted strict conservative treatment. There were no differences between these two groups in early mortality and complications. However, the late mortality of patients in the CG was significantly higher than for patients in the OG. A maximum aortic diameter in the ascending aorta and aortic arch $\geq 45 \mathrm{~mm}$ and maximum thickness of IMH in the same section $\geq 8 \mathrm{~mm}$ were risk factors for $\mathrm{IMH}$ related death in patients undergoing conservative treatment.

Conclusions: The mortality associated with emergency surgery for patients with acute type A aortic IMH was satisfactory. In clinical centers with well-established surgical techniques and postoperative management, emergency surgical treatment may provide a better outcome than medical treatment for patients with acute type A aortic IMH.
\end{abstract}

Keywords: Aortic intramural hematoma, Therapeutic management, Surgical treatment, Conservative treatment

\section{Introduction}

Acute aortic intramural hematoma (IMH) is an entity within the spectrum of acute aortic syndrome (AAS), in which a hematoma develops in the media of the aortic wall in the absence of a false lumen and intimal tear [1]. The natural history of acute aortic IMH is highly variable,

\footnotetext{
*Correspondence: fanruixin@163.com

${ }^{\dagger}$ Jue Yang, Changjiang Yu and Xin Li have contributed equally to this work ${ }^{1}$ Department of Cardiac Surgery, Guangdong Cardiovascular Institute, Guangdong Provincial Key Laboratory of South China Structural Heart

Disease, Guangdong Provincial People's Hospital, Guangdong Academy of Medical Sciences, 96 Dongchuan Road, Guangzhou, Guangdong, China

Full list of author information is available at the end of the article The corresponding authors are Miaoxian Fang and Ruixin Fan
}

including hematoma resolution, progression to aortic dissection, aneurysm formation, and aortic rupture [2].

The proper therapeutic management for acute aortic $\mathrm{IMH}$ is still controversial, especially for type A aortic IMH. In Western countries, the management of acute type A aortic IMH is emergency or urgent surgery. However, in Japan and Korea, there has been a growing trend towards conservative treatment without surgery for acute type A aortic IMH [2-7]. Thus, we need additional longterm and large scale research to make optimal therapeutic management decisions for these patients. In this report, we retrospectively reviewed our experience in the treatment of acute type A IMH and compared the outcomes for emergency or urgent surgery with conservative treatment for patients with this disease. 


\section{Patients and methods Study patients}

From January 2015 to December 2018, 124 consecutive patients were diagnosed with acute type A aortic IMH in our hospital. In our study, aortic IMH was defined as the presence of a circular or crescent shaped thickening of the aortic wall $(\geq 5 \mathrm{~mm})$ in the absence of detectable blood flow inside(a high attenuation area not enhanced after contrast medium administration) on multidetector computed tomography(MDCT), and accompanied by a clinical presentation compatible with acute aortic syndrome. The acute phase refers to 14 days from onset of this disease. The study was approved by the research ethics committee of Guangdong Provincial People's Hospital and informed consent was obtained from all participants.

\section{Management and follow-up}

In our emergency room, all patients with suspected AAS received a contrast MDCT and transthoracic echocardiography. The MDCT images were used to confirm a diagnosis of acute type A aortic IMH. The aortic IMH lesion type was classified based on the initial diagnosis (type A or type B) using the earliest imaging data from MDCT and the initial diagnosis was not changed even if the lesion type changed (from type B to type A).

Following a comprehensive assessment, patients with shock (requiring vasoactive drugs), severe hypoxemia (requiring tracheal intubation), moderate or massive pericardial effusion, severe aortic valve regurgitation (AR), acute myocardial infarction and other severe malperfusion syndrome were selected for emergency surgery. Emergency surgery was also performed on patients if the maximum aortic diameter in the ascending aorta and aortic arch was more than $50 \mathrm{~mm}$ or the maximum thickness of IMH in the same section was more than $11 \mathrm{~mm}$. Other patients received conservative treatment. They were initially treated with an intravenously administered of beta-blocker, calcium antagonist and pain relieving drug to maintain systolic blood pressure to less than $120 \mathrm{mmHg}$. Three days later, if these patients were in stable condition, they would receive these medications orally.

After discharge, all patients received strict followed up at one month, three months, six months, 12 months and then yearly. This included a contrast MDCT and transthoracic echocardiography at each follow-up visit. If any patients who received conservative treatment initially were found to meet the above surgical indications at follow-up, emergency surgery was performed.

Patients in the surgical and conservative treatment groups were compared in terms of in-hospital mortality, morbidity, and long-term outcomes.

\section{Surgical procedure}

Usually, cardiopulmonary bypass was implemented via right axillary arterial cannulation. If the right axillary artery was anomalous or too small, the arterial cannulation was performed through the aortic arch with the help of transesophageal echocardiography. Two venous cannulas were respectively inserted into the superior vena cava and inferior vena cava. All patients were cooled to 24 degree centigrade(rectal temperature) and antegrade selective cerebral perfusion was performed through the right axillary arterial cannulation or left carotid arterial cannulation. All patients received ascending aortic replacement and total aortic arch replacement with an intraoperative stent inserted into the descending aorta. If aortic valve disease or dilation of the aortic sinus was present, a Bentall procedure was performed at the same time. For some patients with coronary heart disease or other valvular heart disease, coronary artery bypass grafting and cardiac valvular surgery was performed concomitantly.

\section{Statistical analysis}

Statistical analysis was performed using SPSS version 25.0 for Windows (SPSS, Chicago, IL). Continuous variables were expressed as medians, means \pm standard deviations, or both. Categorical variables were expressed as frequencies and percentages. Univariate analysis and logistic regression analysis were performed to identify risk factors for death in patients with conservative treatment. For counting variables, we used the $t$ test to perform univariate analysis. For categorical variables, we used the $\chi^{2}$ test to perform univariate analysis. If the $\mathrm{P}$ value was less than 0.1 , the variable would be included in the logistic regression analysis. If the P-value in logistic regression analysis was less than 0.05 , we considered the variable to be a risk factor for postoperative death. Survival curves were generated via the Kaplan-Meier method with significant differences assessed for time-toevent data using log-rank tests.

\section{Results}

This study included 110 males and 14 females. Mean age was $53.5 \pm 13.1$ years, ranging from 26 to 79 years. Time from onset of disease to diagnosis was $18.3 \pm 6.7 \mathrm{~h}$, ranging from 12 to $36 \mathrm{~h}$. All patients had a history of hypertension and no patients with Marfan syndrome were included. Ten patients had coronary artery disease and seven patients had a history of stroke. A total of $8.1 \%$ and $10.5 \%$ of the patients had hyperlipidemia and diabetes, respectively. All 124 patients received transthoracic echocardiography and the grades of aortic valve regurgitation were as follows: 11 none, 53 mild, 47 moderate, 
13 severe. All patients received contrast MDCT and 22 patients were found to have massive pericardial effusion. In addition, 38 patients exhibited moderate pericardial effusion and 34 patients had small pericardial effusion. Through the MDCT, we also collected imaging data for every patient. The maximum aortic diameter in the ascending aorta and aortic arch was $45.3 \pm 6.5 \mathrm{~mm}$, ranging from $35.8 \mathrm{~mm}$ to $55.2 \mathrm{~mm}$. The maximum thickness of IMH in the ascending aorta and aortic arch was $7.6 \pm 4.1 \mathrm{~mm}$, ranging from $5.0 \mathrm{~mm}$ to $15.4 \mathrm{~mm}$. Before our treatment, nine patients suffered from shock and needed vasoactive drugs, while six patients had severe hypoxemia and underwent tracheal intubation. The incidences of acute myocardial infarction and acute renal failure were $3(2.4 \%)$ and $5(4.0 \%)$, respectively.

According to our indications for emergency surgery, in all 124 patients, $83(66.9 \%)$ patients accepted emergency surgery and 41 (33.1\%) patients accepted strict conservative treatment. These patients were divided into two groups: the operation group (OG) and the conservative treatment group (CG). There were no differences between these two groups in terms of age, gender, time between onset and diagnosis, coronary artery disease, diabetes, hyperlipidemia or history of stroke (Table 1). All patients in the OG received ascending aortic replacement and total aortic arch replacement with an intraoperative stent inserted into the descending aorta. Ten also received a Bentall procedure. Two patients received coronary artery bypass grafting due to coronary artery disease. Two other patients received mitral valve repair due to severe mitral valve regurgitation.

\section{Early outcomes}

In the operation group, six patients died before hospital discharge. The early mortality was $7.2 \%(6 / 83)$. The causes of death included heart failure (one patient), cerebral hemorrhage (one patient), sepsis (two patients) and multiple organ dysfunction syndrome (two patients). The incidence of postoperative heart failure, cerebrovascular

Table 1 Clinical characteristics of patients

\begin{tabular}{llll}
\hline & OG(n=83) & CG(n=41) & P value \\
\hline Age (y) & $54.7 \pm 14.2$ & $51.1 \pm 11.5$ & 1.000 \\
Gender (male/female) & $73 / 10$ & $37 / 4$ & 0.938 \\
$\begin{array}{l}\text { Time between onset and } \\
\quad \text { diagnosis (h) }\end{array}$ & $17.7 \pm 6.3$ & $19.4 \pm 7.0$ & 1.000 \\
Coronary artery disease & 6 & 4 & 0.892 \\
Diabetes & 8 & 5 & 0.900 \\
Hyperlipidemia & 7 & 3 & 1.000 \\
History of stroke & 5 & 2 & 1.000 \\
\hline
\end{tabular}

OG operation group, $C G$ conservative treatment group event, paraplegia, pneumonia, multiple organ dysfunction syndrome and renal failure were 3 (3.6\%), 5 (6.0\%), 2(2.4\%), 4 (4.8\%), $2(2.4 \%)$ and $3(3.6 \%)$ patients, respectively. In the conservative treatment group, three patients died before hospital discharge. The early mortality was $7.3 \%(3 / 41)$. The causes of death included cardiac tamponade (two patients) and acute myocardial infarction (one patient). Otherwise, two patients suffered a cerebrovascular event and two other patients had pneumonia. No significant differences between two groups in terms of early outcomes were identified (Table 2).

\section{Late outcomes}

After discharge, all surviving patients underwent followup at one month, three months, six months, 12 months and yearly. The mean follow-up time was $3.3 \pm 1.1$ years, ranging from 2 to 5 years. In the operation group, one patient died from a descending aortic rupture one year later and another patient died in a traffic accident. The total IMH related mortality was $8.5 \%(7 / 82)$ due to one censored data. In the conservative treatment group, four patients died of thoracic aortic rupture, two patients died of cardiac tamponade and one patient died of lymphoma. Otherwise, during follow-up, two patients in the CG were found to have dilation of the ascending aorta and in both cases, their maximum ascending aortic diameter was more than $50 \mathrm{~mm}$. They both underwent emergency surgery. The total IMH related mortality was $22.5 \%(9 / 40)$ due to one censored data. There was a significant difference between the two groups in total IMH related mortality $(\mathrm{P}=0.032)$. There was also a significant difference in actuarial survival at 5 years, which was $90.4 \%$ in the OG and $74.3 \%$ in the CG (Fig. 1).

The mortality of patients in the CG was significantly higher than patients in the OG. Therefore, univariate analysis and logistic regression analysis were performed to identify risk factors for IMH related death in patients with conservative treatment. At first, we

Table 2 Hospital mortality and morbidity

\begin{tabular}{llll}
\hline & OG $(\mathbf{n}=\mathbf{8 3})$ & CG $(\mathbf{n}=\mathbf{4 1})$ & P value \\
\hline Heart failure & 3 & 0 & 0.550 \\
Cerebrovascular event & 5 & 2 & 1.000 \\
Pneumonia & 4 & 2 & 1.000 \\
MODS & 2 & 0 & 1.000 \\
Renal failure & 3 & 0 & 0.550 \\
Cardiac tamponade & 0 & 2 & 0.108 \\
Acute myocardial infarction & 0 & 1 & 0.331 \\
Hospital mortality & 6 & 3 & 1.000 \\
\hline
\end{tabular}

OG operation group, $C G$ conservative treatment group, MODS multiple organ dysfunction syndrome 


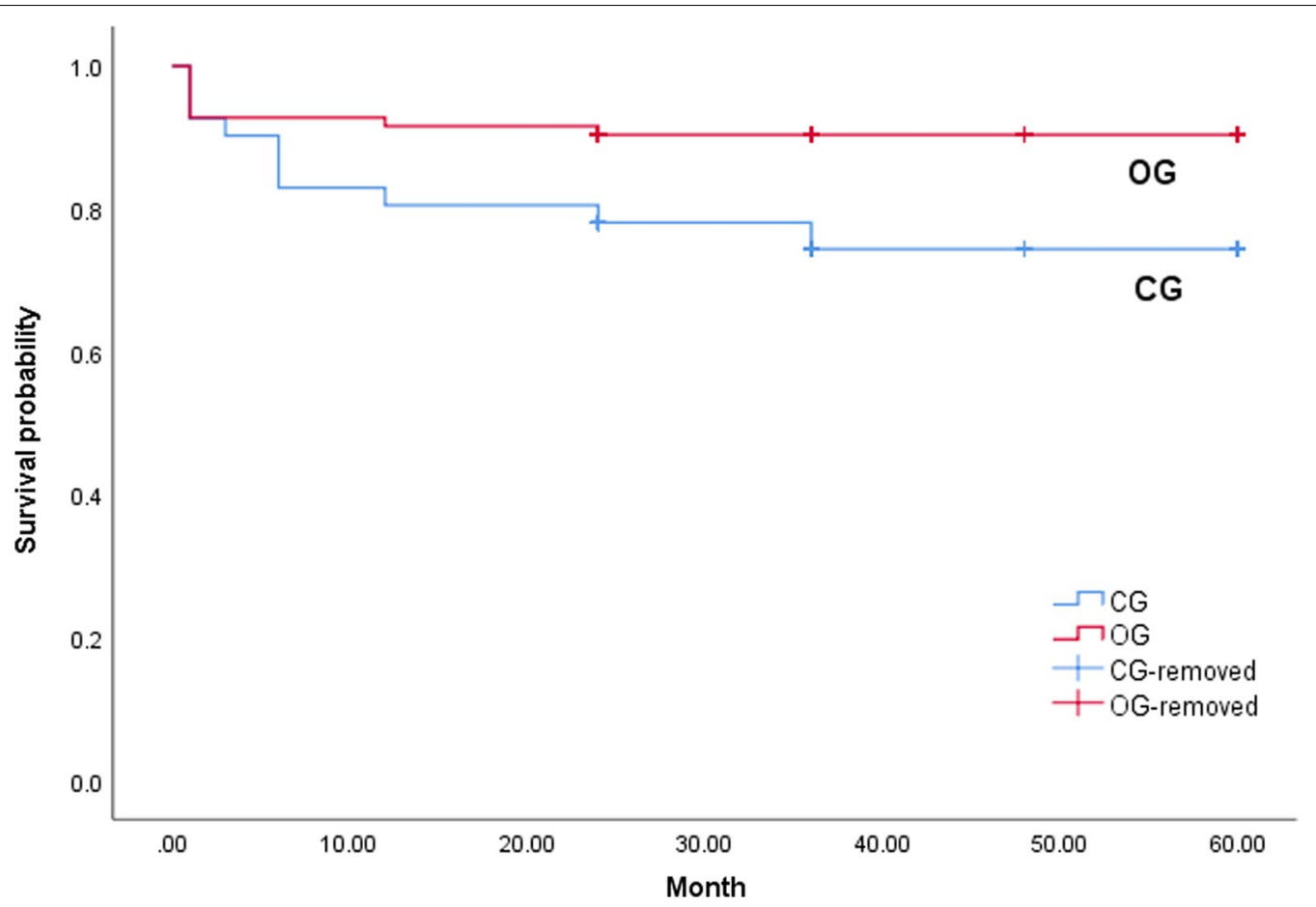

Fig. 1 Actuarial survival curves. There was significant difference in actuarial survival at 5 years, which was $90.4 \%$ in the OG and $74.3 \%$ in the CG $(P=0.010)$

chose age, maximum aortic diameter in the ascending aorta and aortic arch, maximum thickness of IMH in the ascending aorta and aortic arch, aortic valve regurgitation, pericardial effusion, coronary artery disease and diabetes as proper risk factors related to death and performed univariate analysis ( $x^{2}$ test) one-by-one. The results of univariate analysis are shown in Table 3.

On the basis of univariate analysis, we singled out maximum aortic diameter in the ascending aorta and aortic arch and maximum thickness of IMH in the same section to undertake binary logistic regression analysis. The results of binary logistic regression analysis are shown in Table 4.

On the basis of binary logistic regression analysis, we found that maximum aortic diameter in the ascending aorta and aortic arch and maximum thickness of IMH in the same section were significantly associated with IMH related death in patients with conservative treatment. In the CG, the IMH related mortality of patients with maximum aortic diameter in the ascending aorta and aortic arch $\geq 45 \mathrm{~mm}$ or maximum thickness of IMH in the same section $\geq 8 \mathrm{~mm}$ was significantly higher than that of other patients. They were risk factors for IMH related death in patients with conservative treatment.
Table 3 Results of univariate analysis

\begin{tabular}{|c|c|c|}
\hline & Death/totality & P value \\
\hline Age & & 0.490 \\
\hline$\geq 60 y$ & $2 / 15$ & \\
\hline$<60 y$ & $7 / 25$ & \\
\hline Maximum aortic diameter ${ }^{\mathrm{a}}$ & & 0.000 \\
\hline$\geq 45 \mathrm{~mm}$ & $6 / 8$ & \\
\hline$<45 \mathrm{~mm}$ & $3 / 32$ & \\
\hline Maximum thickness of aortic $\mathrm{IMH}^{\mathrm{a}}$ & & 0.049 \\
\hline$\geq 8 \mathrm{~mm}$ & $5 / 10$ & \\
\hline$<8 \mathrm{~mm}$ & $4 / 30$ & \\
\hline Aortic valve regurgitation & & 0.284 \\
\hline Moderate & $5 / 14$ & \\
\hline None and mild & $4 / 26$ & \\
\hline Pericardial effusion & & 0.238 \\
\hline Small & $7 / 22$ & \\
\hline None & $2 / 18$ & \\
\hline Coronary artery disease & & 1.000 \\
\hline Yes & $1 / 4$ & \\
\hline No & $8 / 36$ & \\
\hline Diabetes & & 0.668 \\
\hline Yes & $2 / 5$ & \\
\hline No & $7 / 35$ & \\
\hline
\end{tabular}


Table 4 The results of binary logistic regression analysis

\begin{tabular}{|c|c|c|c|c|c|}
\hline & \multirow[t]{2}{*}{ Death/totality } & \multirow[t]{2}{*}{ Odds ratio (OR) } & \multicolumn{2}{|c|}{ 95\% Confidence Interval (Cl) } & \multirow[t]{2}{*}{$\mathbf{P}$} \\
\hline & & & Lower limit & Upper limit & \\
\hline Maximum aortic diameter ${ }^{\mathrm{a}}$ & & 15.219 & 6.239 & 37.126 & 0.000 \\
\hline$\geq 45 \mathrm{~mm}$ & $6 / 8$ & & & & \\
\hline$<45 \mathrm{~mm}$ & $3 / 32$ & & & & \\
\hline Maximum thickness of aortic $\mathrm{IMH}^{\mathrm{a}}$ & & 23.408 & 10.618 & 51.603 & 0.000 \\
\hline$\geq 8 \mathrm{~mm}$ & $5 / 10$ & & & & \\
\hline$<8 \mathrm{~mm}$ & $4 / 30$ & & & & \\
\hline
\end{tabular}

a: in the ascending aorta and aortic arch

Abbreviations: IMH = intramural hematoma

\section{Discussion}

The natural history of acute type A aortic intramural hematoma is extremely variable and controversy still exists regarding whether emergency surgery should be carried out for all acute type A aortic IMH [2-7]. In the European (ESC-AD 2014 guidelines) and American (ACCF-AD 2010 guidelines) guidelines, emergency surgery is recommended for all acute type A aortic IMH [1, 8]. In the Asia Task Force practice guidelines for aortic disease (JCS-AD 2011 guidelines), conservative treatment is recommended for an acute type A aortic IMH in patients with a hematoma thickness $<11 \mathrm{~mm}$ and an aortic diameter $<50 \mathrm{~mm}$ [9]. However, the data supporting the Asian guidelines mainly came from studies performed in Japan and South Korea and data from the Chinese population is lacking. Moreover, studies supporting both the Eastern and Western guidelines were not randomized trials, and the numbers of enrolled patients were fewer than one hundred even in multicenter studies $[6,10-13]$. These numbers are actually too low to provide sufficient statistical power. The data presented in this study may greatly contribute to improvements in practice guidelines for acute type A aortic IMH.

In the present study, our surgical indications for patients with acute type A aortic IMH were based on JCS-AD 2011 guidelines but also included moderate or massive pericardial effusion and severe aortic valve regurgitation into indications of emergency surgery per our clinical experience. Even though our indications for medical treatment were stricter than those in the JCSAD 2011 guidelines, the mortality of patients with only medical treatment was significantly higher in followup than patients treated with surgery. For patients with acute type A aortic IMH, even though contrast MDCT or transthoracic echocardiography on admission or in follow-up did not show any false lumen or intimal tear, blood oozing may have developed gradually and the firmness of the aortic wall may have weakened, allowing cardiac tamponade and aortic rupture to happen. Four patients who underwent medical treatment died of cardiac tamponade and their $\mathrm{CT}$ and echocardiography only showed a small pericardial effusion. Four other patients in the conservative group died from an aortic rupture. However, in their contrast MDCT, all had a maximum aortic diameter in the ascending aorta and aortic arch of less than $50 \mathrm{~mm}$ and the maximum thickness of IMH in the same section was less than $11 \mathrm{~mm}$. On the other hand, the mortality associated with surgery in acute type A aortic IMH has rapidly decreased due to improvements in surgical techniques and postoperative management. In our study, the total IMH related mortality was only $8.5 \%$ yet 10 years ago, this rate ranged from 12.5 to $39.1 \%[6,14]$. Therefore, nowadays, even when contrast MDCT and transthoracic echocardiography reveal a type A aortic IMH, performing surgery as soon as possible is considered to be a main factor to improving prognosis. Mitsumasa and colleagues [15] have also reported poor outcomes following medical treatment of acute type A aortic IMH. In their research, the freedom from aortic event was only $46.8 \%$ at 10 years. They thought emergency surgical treatment may provide a better outcome than medical treatment. In our study, patients in the CG had "much better aorta" than patients in the OG. However, they showed very bad outcomes in terms of aortic events. In the CG, nine patients died of aortic events. In the OG, only one patients suffered from descending rupture. We thought for most patients with type A aortic IMH emergency surgery may be the best choice.

Song and colleagues [6] have reported that in patients of type A aortic IMH with medical treatment, initial aorta diameter and hematoma thickness were independent predictors for adverse clinical events including death and delayed surgery. In our study, we also found that the maximum aortic diameter in the ascending aorta and aortic arch and the maximum thickness of IMH in the same section were risk factors for IMH 
related death in patients with conservative treatment. In JCS-AD 2011 guidelines, the best cutoff values were $50 \mathrm{~mm}$ in aortic diameter and $11 \mathrm{~mm}$ in hematoma thickness. However, in our study, the IMH related mortality of patients with maximum aortic diameter in the ascending aorta and aortic arch $\geq 45 \mathrm{~mm}$ or maximum thickness of IMH in the same section $\geq 8 \mathrm{~mm}$ was significantly higher than that of other patients. Thus, we thought we should make more stringent indications of medical treatment in patients with acute type A aortic IMH.

Finally, in the CG, 30 patients survived. In the follow-up period, 4 suffered complications of pneumonia and heart failure. During the follow-up for the OG, no patient showed these complications. This may be another advantage of surgical treatment. In the CG, bed rest is necessary and important for antihypertensive therapy, particularly in critically sick patients. However, it is associated with risk factors of depression, pneumonia and heart failure [16]. On the other hand, in the OG, the patients could eat and drink on postoperative days. Furthermore, they could take a walk and even work 3 months later because the lesion in the ascending aorta and aortic arch had already been repaired. Therefore, emergency surgery could enable much easier management of patients post-surgery and improve their quality of life compared with medical management.

Our study has several limitations, including all of the limitations related to a retrospective study from a single institution. Furthermore, the small number of patients and the short follow-up time limit the statistical power of the study. More refined studies are necessary in the future to completely resolve the controversy as to the best treatment of acute type A aortic IMH.

\section{Conclusions}

The mortality of emergency surgery for patients with acute type A aortic IMH was satisfactory. In clinical centers with well-established surgical techniques and postoperative management, emergency surgical treatment may provide a better outcome than medical treatment for patients with acute type A aortic IMH.

\section{Abbreviations \\ CG: Conservative treatment group; IMH: Intramural hematoma; MODS: Multi- ple organ dysfunction syndrome; OG: Operation group.}

\section{Acknowledgements}

Not applicable.

\section{Authors' contributions}

$J Y, C Y$ and $X L$ wrote the main manuscript text. ZC and FX prepared Tables 1, 2, 3 and 4. TS prepared Fig. 1. JK and MF revised the manuscript. RF was the manager of the whole study. All authors read and approved the final manuscript.

\section{Funding}

Supported by National Key Research and Development Program of China(2017YFC1308003) and Guangzhou Science and Technology Program Key Projects(202102020160). The funding body supported collection, professional advice from a statistician in Southern Medical University for suitable statistical analyses and from Wiley Editing Service for the quality of English.

\section{Availability of data and materials}

The datasets used and/or analysed during the current study available from the corresponding author on reasonable request.

\section{Declarations}

\section{Ethics approval and consent to participate}

This retrospective study was approved by the ethics committee of Guangdong Provincial People's Hospital, Guangdong Academy of Medical Sciences, Guangzhou, China (No.GDREC2018322H(R2)). Written and informed consent was obtained from the patients included in this study.

\section{Consent for publication \\ Not applicable.}

\section{Competing interests}

The authors declare that they have no competing interests.

\section{Author details}

${ }^{1}$ Department of Cardiac Surgery, Guangdong Cardiovascular Institute, Guangdong Provincial Key Laboratory of South China Structural Heart Disease, Guangdong Provincial People's Hospital, Guangdong Academy of Medical Sciences, 96 Dongchuan Road, Guangzhou, Guangdong, China. ${ }^{2}$ Department of Cardiovascular Surgery, Guangzhou First People' Hospital, Guangzhou, China.

Received: 20 March 2021 Accepted: 3 June 2021

Published online: 10 June 2021

\section{References}

1. Erbel R, Aboyans V, Boileau C, et al. ESC Committee for Practice Guidelines, 2014 ESC guidelines on the diagnosis and treatment of aortic diseases: document covering acute and chronic aortic diseases of the thoracic and abdominal aorta of the adult. The Task Force for the Diagnosis and Treatment of Aortic Diseases of the European Society of Cardiology (ESC) . Eur Heart J. 2014;35:2873-926.

2. Song JK. Update in acute aortic syndrome: intramural hematoma and incomplete dissection as new disease entities. J Cardiol. 2014;64:153-61.

3. Pelzel JM, Braverman AC, Hirsch AT, et al. International heterogeneity in diagnostic frequency and clinical outcomes of ascending aortic intramural hematoma. J Am Soc Echocardiogr. 2007;20:1260-8.

4. Evangelista A, Mukherjee D, Mehta RH, et al. Acute intramural hematoma of the aorta: a mystery in evolution. Circulation. 2005;111:1063-70.

5. Kitai T, Kaji S, Yamamuro A, et al. Clinical outcomes of medical therapy and timely operation in initially diagnosed type A aortic intramural hematoma: a 20-year experience. Circulation. 2009;120(Suppl 11):S292-8.

6. Song JK, Yim JH, Ahn JM, et al. Outcomes of patients with acute type A aortic intramural hematoma. Circulation. 2009;120:2046-52.

7. Harris KM, Braverman AC, Eagle KA, et al. Acute aortic intramural hematoma: an analysis from the International Registry of Acute Aortic Dissection. Circulation. 2012;126(Suppl 11):S91-6.

8. Hiratzka LF, Bakris GL, Beckman JA, et al. 2010 ACCF/AHA/AATS/ACR/ASA/ SCA/SCAI/SIR/STS/SVM guidelines for the diagnosis and management of patients with Thoracic Aortic Disease: a report of the American College of Cardiology Foundation/American Heart Association Task Force on Practice Guidelines, American Association for Thoracic Surgery, American College of Radiology, American Stroke Association, Society of Cardiovascular Anesthesiologists, Society for Cardiovascular Angiography and Interventions, Society of Interventional Radiology, Society of Thoracic Surgeons, and Society for Vascular Medicine. Circulation. 2010;121(13):e266-369. 
9. JCS Joint Working Group. Guidelines for diagnosis and treatment of aortic aneurysm and aortic dissection (JCS 2011) - Digest Version. Circ J. 2013;77:789-828.

10. Kaji S, Akasaka T, Horibata Y, et al. Long term prognosis of patients with type A aortic intramural hematoma. Circulation. 2002;106(12 Suppl 1):1248-52.

11. Moizumi Y, Komatsu T, Motoyoshi N, Tabayashi K. Management of patients with intramural hematoma involving the ascending aorta. J Thorac Cardiovasc Surg. 2002;124:918-24.

12. Evangelista A, Dominguez R, Sebastia C, et al. Prognostic value of clinical and morphologic findings in short-term evolution of aortic intramural hematoma: therapeutic implications. Eur Heart J. 2004;25:81-7.

13. Song JM, Kim HS, Song JK. Usefulness of the initial noninvasive imaging study to predict the adverse outcomes in the medical treatment of acute type A aortic intramural hematoma. Circulation. 2003;108(Suppl 1):324-8.
14. Ferrera C, Vilacosta I, Gómez-Polo, et al. Evolution and prognosis of intramural aortic hematoma. Insights from a midterm cohort study. Int J Cardiol. 2017;249:410-3.

15. Mitsumasa H, Hiroaki, , et al. Optimal treatment strategy for type A acute aortic dissection with intramural hematoma. J Thorac Cardiovasc Surg. 2014;147:307-11.

16. Niino T, Hata M, Sezai A, et al. Optimal clinical pathway for the patient with type B acute aortic dissection. Circ J. 2009;73:264-8.

\section{Publisher's Note}

Springer Nature remains neutral with regard to jurisdictional claims in published maps and institutional affiliations.
Ready to submit your research? Choose BMC and benefit from:

- fast, convenient online submission

- thorough peer review by experienced researchers in your field

- rapid publication on acceptance

- support for research data, including large and complex data types

- gold Open Access which fosters wider collaboration and increased citations

- maximum visibility for your research: over $100 \mathrm{M}$ website views per year

At BMC, research is always in progress.

Learn more biomedcentral.com/submissions 